\title{
Use of radio occultation to probe the high-latitude ionosphere
}

\author{
A. J. Mannucci, B. T. Tsurutani, O. Verkhoglyadova, A. Komjathy, and X. Pi \\ Jet Propulsion Laboratory, California Institute of Technology, Pasadena, CA, USA \\ Correspondence to: A. J. Mannucci (tony.mannucci@jpl.nasa.gov) \\ Received: 02 November 2014 - Published in Atmos. Meas. Tech. Discuss.: 23 February 2015 \\ Revised: 19 June 2015 - Accepted: 22 June 2015 - Published: 16 July 2015
}

\begin{abstract}
We have explored the use of COSMIC data to provide valuable scientific information on the ionospheric impacts of energetic particle precipitation during geomagnetic storms. Ionospheric electron density in the E region, and hence ionospheric conductivity, is significantly altered by precipitating particles from the magnetosphere. This has global impacts on the thermosphere-ionosphere because of the important role of conductivity on high-latitude Joule heating. Two high-speed stream (HSS) and two coronal mass ejection (CME) storms are examined with the COSMIC data. We find clear correlation between geomagnetic activity and electron density retrievals from COSMIC. At nighttime local times, the number of profiles with maximum electron densities in the E layer (below $200 \mathrm{~km}$ altitude) is well correlated with geomagnetic activity. We interpret this to mean that electron density increases due to precipitation are captured by the COSMIC profiles. These "E-layer-dominant ionosphere" (ELDI) profiles have geomagnetic latitudes that are consistent with climatological models of the auroral location. For the two HSS storms that occurred in May of 2011 and 2012, a strong hemispheric asymmetry is observed, with nearly all the ELDI profiles found in the Southern, less sunlit, Hemisphere. Stronger aurora and precipitation have been observed before in winter hemispheres, but the degree of asymmetry deserves further study. For the two CME storms, occurring in July and November of 2012, large increases in the number of ELDI profiles are found starting in the storm's main phase but continuing for several days into the recovery phase. Analysis of the COSMIC profiles was extended to all local times for the July 2012 CME storm by relaxing the ELDI criterion and instead visually inspecting all profiles above $50^{\circ}$ magnetic latitude for signatures of precipitation in the E region. For 9 days during the July 2012 period, we find a signature of precipitation occurs nearly uniformly in local time, although the magnitude of electron density increase
\end{abstract}

may vary with local time. The latitudinal extent of the precipitation layers is generally consistent with auroral climatology. However, after the storm main phase on 14 July 2012 the precipitation tended to be somewhat more equatorward than the climatology (by about $5-10^{\circ}$ latitude) and equatorward of the auroral boundary data acquired from the SSUSI sensor onboard the F18 DMSP satellite. We conclude that, if analyzed appropriately, high-latitude COSMIC profiles have the potential to contribute to our understanding of MI coupling processes and extend and improve existing models of the auroral region.

\section{Introduction}

The high-latitude ionosphere, encompassing the auroral and polar cap regions, is an area of important processes related to magnetosphere-ionosphere coupling that has global impacts on the thermosphere-ionosphere system. New scientific results have come from coordinated use of ground and space observations. The high latitudes are reasonably well instrumented with ground-based data, including radars, imagers and GPS receivers (total electron content) that cover wide geographic areas. Operational satellite missions are providing data on a continuous basis from high latitudes.

Despite these resources, critical quantities are only observed indirectly or sparsely, such as the changes in highlatitude conductivity resulting from energetic particle precipitation (EPP). Ionospheric electron density, and hence conductivity, is significantly altered by precipitating particles from the magnetosphere, which has global impacts because of the important role of conductivity on high-latitude Joule heating (Cole, 1962; Wiltberger et al., 2004). The community has devoted significant resources to developing climatological models that provide quantitative information on auroral 
zone precipitation and the resultant conductivities (Robinson et al., 1987; Fuller-Rowell and Evans, 1987; Zhang and Paxton, 2008). A lack of observations has hampered more detailed understanding of these processes, because it requires simultaneous observations in the lower ionosphere ( $\sim 120 \mathrm{~km}$ altitude) where conductivity can increase substantially and measurements of particle precipitation usually obtained from satellites at higher altitudes (e.g., $\sim 800 \mathrm{~km}$ altitude). High-latitude patterns of particle precipitation driven by satellite data have been developed and are widely used as inputs to global models of the thermosphere-ionosphere (Fuller-Rowell and Evans, 1987). The resulting conductivity changes have been developed using observations and theory that relates average particle energy to conductivity changes at specific locations and times (Robinson et al., 1987). The reliance on climatological perspectives suggests that new insights are possible by significantly increasing the observations in one domain or the other.

The Constellation Observing System for Meteorology Ionosphere and Climate (COSMIC) is a constellation of six orbiting satellites with onboard GPS receivers that acquire total electron content data in limb viewing geometries as the GPS satellites occult behind Earth from the perspective of the low-Earth orbiters. Using the assumption of local spherical symmetry near the ray path tangent point, profiles of electron density versus altitude are retrieved. Although local spherical symmetry is not realistic at high latitudes, COSMIC data can be an extremely sensitive monitor of narrow ( $\sim$ few kilometers in altitude) electron density layers due to the limb geometry. In this paper, we explore the potential of COSMIC to provide new information on the ionospheric consequences of energetic particle precipitation by analyzing to what degree COSMIC data can reveal impacts of EPP. We find prominent $\mathrm{E}$ layer signatures of EPP that are well correlated with geomagnetic activity during the storms studied. The broad high-latitude coverage of COSMIC observations suggests significant new information is possible with the constellation, which will only increase with follow-on constellations that acquire significantly more data. In this way, improved representations of how EPP impact the ionosphere are possible. Ultimately, these representations can be used to improve space weather forecasting.

In the next section we describe the COSMIC observations and how they are used to detect EPP signatures in the upper atmosphere. We then present results for four geomagnetic storms, originating from both high-speed streams (HSSs), and coronal mass ejections (CMEs). The results are then discussed and a conclusion and suggestions for further work follow.

\section{Observations}

Each COSMIC satellite carries a dual-frequency GPS receiver and two antennas that acquire and track GPS satellites that are in front of and behind the satellite (Fong et al.,
2011; Rocken et al., 2000). The total electron content (TEC) between satellite and transmitter is derived by differencing the carrier phase and pseudorange delays measured at the L1 and L2 frequencies (Schreiner et al., 1999; Hajj and Romans, 1998). Subsequent processing requires the calibration of hardware differential delays that bias the TEC (Stephens et al., 2011). Finally, an Abel integral is applied to the TEC data to yield approximate vertical profiles of electron density versus altitude (Hajj and Romans, 1998; Schreiner et al., 1999). Data are obtained at a cadence of $1 \mathrm{~s}$.

Previous studies of COSMIC electron density profiles have revealed good retrieval accuracy (to within $\sim 15 \%$ or so) of the peak electron density (NmF2) (Lei et al., 2007). Accuracy is degraded below the F2 peak due to horizontal variation of electron density along the ray path and other factors (Yue et al., 2010; Nicolls et al., 2009; Hysell, 2007). This study focuses on prominent layer features below the F2 peak density that are likely to be the ionization signatures of EPP. We analyze the presence or absence of a layer at $E$ region altitudes rather than relying on the magnitude of electron density within the $\mathrm{E}$ layer. The emphasis is on detectable layers rather than analyzing the $\mathrm{E}$ region as a whole for this study because detecting plasma density layers is less susceptible to retrieval errors due to the spherical symmetry assumption required by the standard Abel technique. Spatially localized density enhancements caused by layers will create temporary TEC enhancements in the data that are then inverted by the Abel integral transform (Hajj and Romans, 1998). This ensures the existence of a retrieved electron density layer, although with inexact magnitude if there are significant horizontal gradients along the ray path. The paper by Mayer and Jakowski (2009) focuses on multi-year statistics of profiles where the $\mathrm{E}$ region ionization is larger than the $F$ region, which can occur due to EPP. Our emphasis is what occurs during storm periods and how the signatures of EPP vary throughout the storm. This contrasts with previous studies (Wu et al., 2005; Arras et al., 2008) that focus on more typical quiet time conditions.

We focus on the presence of E region layers in the electron density profiles with peak densities below $200 \mathrm{~km}$ altitude. At geomagnetic latitudes above $60^{\circ}$ such layers are likely to be associated with energetic particle precipitation rather than with the sporadic E layer (see Arras et al., 2008). We analyze quiet days preceding the storm periods to control for layers that are not associated with geomagnetic activity. Example daytime and nighttime profiles with and without E layer signatures of precipitation are shown in Fig. 1. We use such profiles to indicate the presence of particle precipitation as described below. A significant advantage of COSMIC for studying the ionospheric consequences of EPP is the improved geographic coverage compared to incoherent scatter radar, although with less information content per profile than radar.

In Fig. 2a-d we have plotted interplanetary parameters for the four geomagnetically active intervals studied in this 


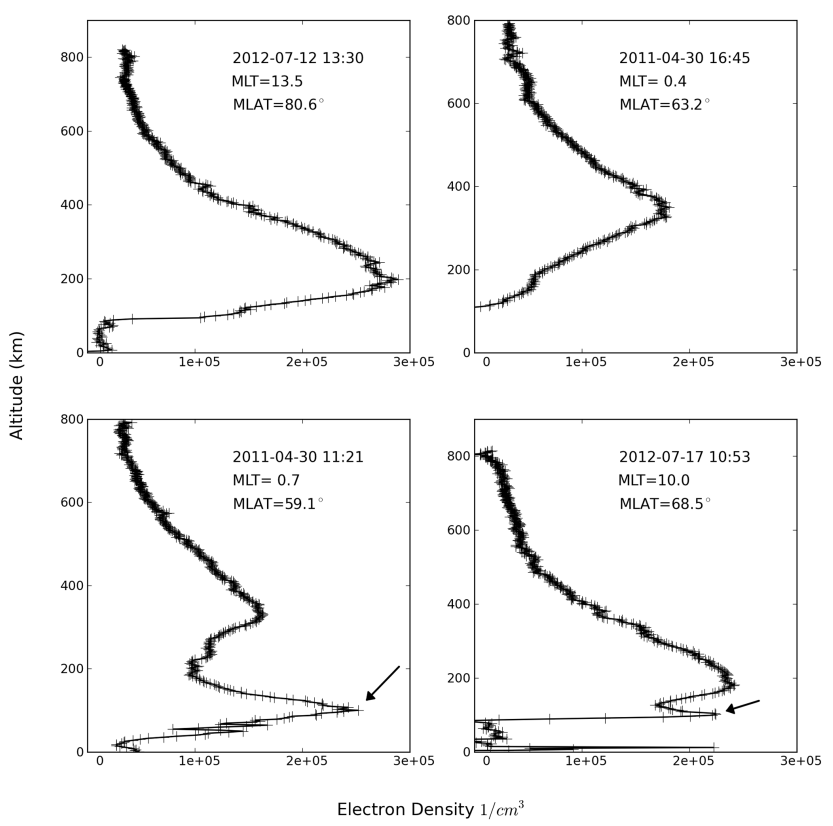

Figure 1. Four representative COSMIC electron density profiles from high latitude. Top: no electron density enhancements; bottom: electron density enhancements, assumed due to energetic particle precipitation, indicated by arrows.

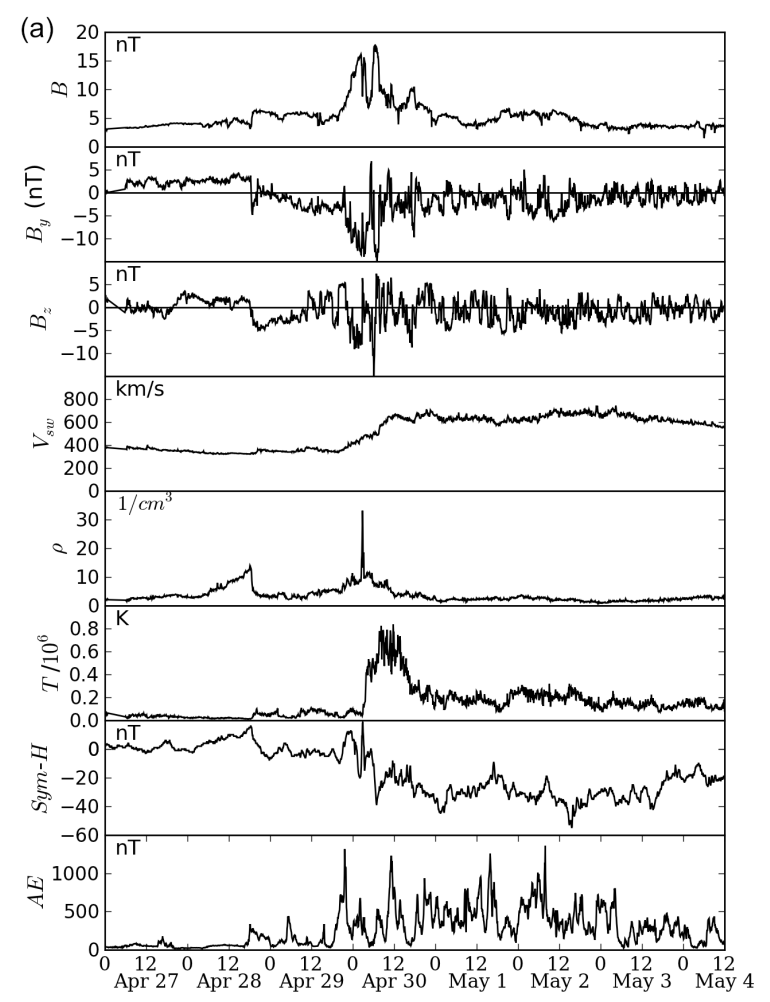

Figure 2a. Solar wind parameters from the OMNI data set for the four events studied is this paper. (a) April 2011; (b) May 2012; (c) July 2012; (d) November 2012.

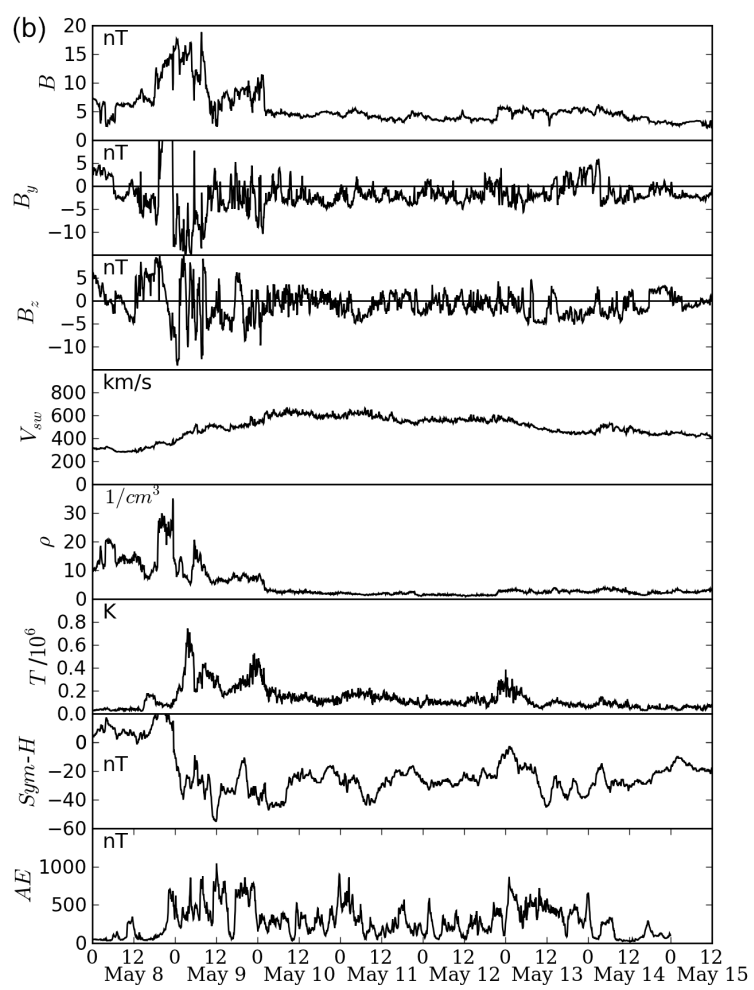

Figure 2b. Continued.

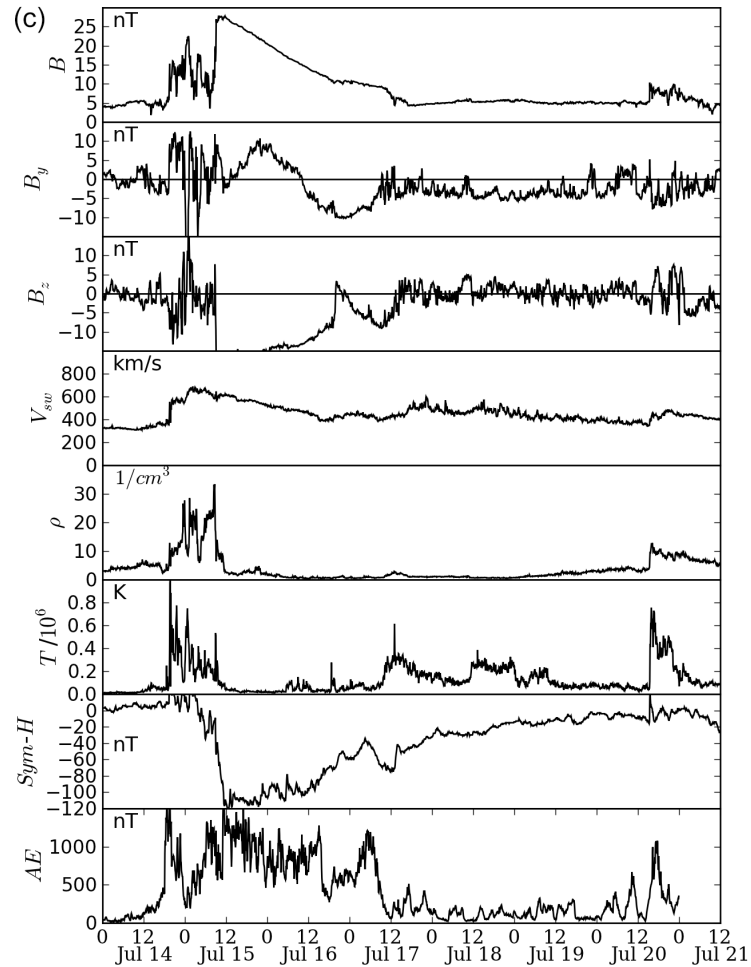

Figure 2c. Continued. 


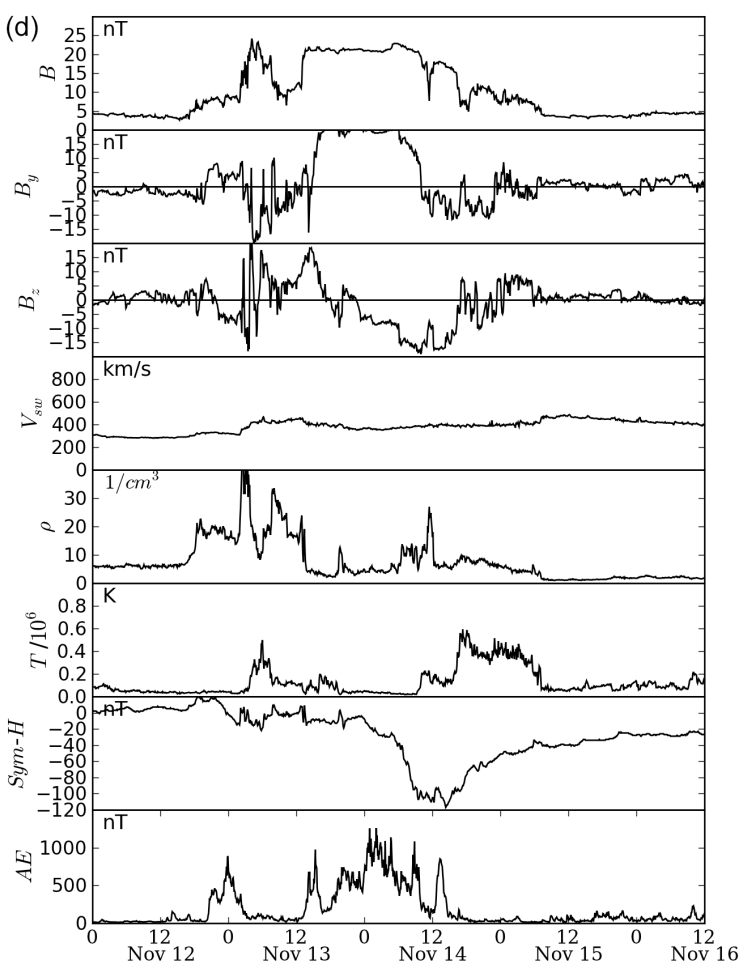

Figure 2d. Continued.

paper, obtained from the OMNI data set at CDAWeb (http: //cdaweb.gsfc.nasa.gov/istp_public/). The two high-speed stream events (Fig. 2a and b) are in April 2011 and May 2012. They show the typical (Tsurutani et al., 2006) interplanetary magnetic field increases due to compression of the plasma at the corotating interaction region (CIR) where the slow and high-speed solar winds interact. Following this increase, there is a period of prolonged geomagnetic activity as indicated by the auroral electrojet (AE) magnetic index, which persists over several days. It has been recently reported that such high-intensity long-duration continuous auroral activity (HILDCAA) periods are associated with an enhancement of relativistic particles in the magnetosphere (Hajra et al., 2014) and in fact that HILDCAAs may be a sufficient condition for the generation of relativistic particles to occur. The non-relativistic particle population, which causes the ionization increases discussed in this paper, may follow similar multi-day association with HILDCAA periods as the relativistic particles.

Interplanetary parameters for the two fast CME events are shown in Fig. 2c and d (July and November 2012, respectively). These are more intense than the CIR-driven geomagnetic storms. These interplanetary CME (ICME) events are characterized by a shock, a sheath and then a magnetic cloud (MC). The shock is characterized by a sudden increase in solar wind velocity, density and temperature and increased magnetic field magnitude. The sheath lies between the shock and the MC, which is a remnant of the CME proper.
In both cases, the southward orientation of the magnetic fields within the MC leads to significant magnetic storms (Dst $<-100 \mathrm{nT}$ ). The auroral activity index reaches higher values during the day of the storm compared to the HSS geomagnetic activity.

The high-speed stream storms had apparent longer durations than the ICME storms did. This is because the southward component of the Alfven waves in the high-speed stream proper continue to pump energy into the magnetosphere well after the CIR has passed (Tsurutani and Gonzalez, 1987).

\section{Remarks on quality control (QC) of COSMIC electron density profiles}

Previous authors have reported on the need to apply QC to the COSMIC electron density profile data (Sheng et al., 2012). This QC is meant to remove gross outliers, not to correct for retrieval errors. It affected over $20 \%$ of the profiles used in the Sheng et al. (2012) study (C. Sheng, private communication, 2012). Analysis at JPL suggests that recent versions of processing at the COSMIC Data Analysis and Archive Center (CDAAC, http://cdaac-www.cosmic. ucar.edu/cdaac/index.html) have largely addressed this problem. An analysis of data showing unphysical profiles suggested that the problem is related to the receiver losing phaselock on the signal in the presence of ionospheric irregularities. The irregularities caused rapid phase excursions of the signal and correspondingly large fluctuations in signal-tonoise ratio, resulting in spurious TEC values. Inverting the TEC using the Abel transform resulted in incorrect electron density retrievals. Data used in this paper were not quality controlled after download from the CDAAC. However, evidence of unphysical data can be found in Fig. 1 (lower right). An apparent "electron density layer" is found at altitudes below $50 \mathrm{~km}$, which is unphysical. Mayer and Jakowski (2009) did not apply QC to earlier versions of the retrievals, apparently without affecting their conclusions. In this work, we did not use the lowest altitudes of the profiles, thus removing the occasional remaining unphysical results.

\section{Results}

To investigate the signature of EPP on COSMIC electron density profiles, we analyzed the altitude of the electron density peak value with altitude. When the peak occurs below $\sim 200 \mathrm{~km}$ altitude, we assume ionization due to precipitation is a dominant factor in the profile. (At geomagnetic latitudes above $60^{\circ}$, we do not consider sporadic $\mathrm{E}$ as a source of $\mathrm{E}$ region electron density layers. See Wu et al., 2005, and Arras et al., 2008.) Mayer and Jakowski (2009) termed such profiles "E-layer-dominated ionosphere" (ELDI), indicating the presence of precipitation. They statistically analyzed such profiles over an extended period comprising several geomagnetic disturbances. The presence of ELDI tends to occur at 


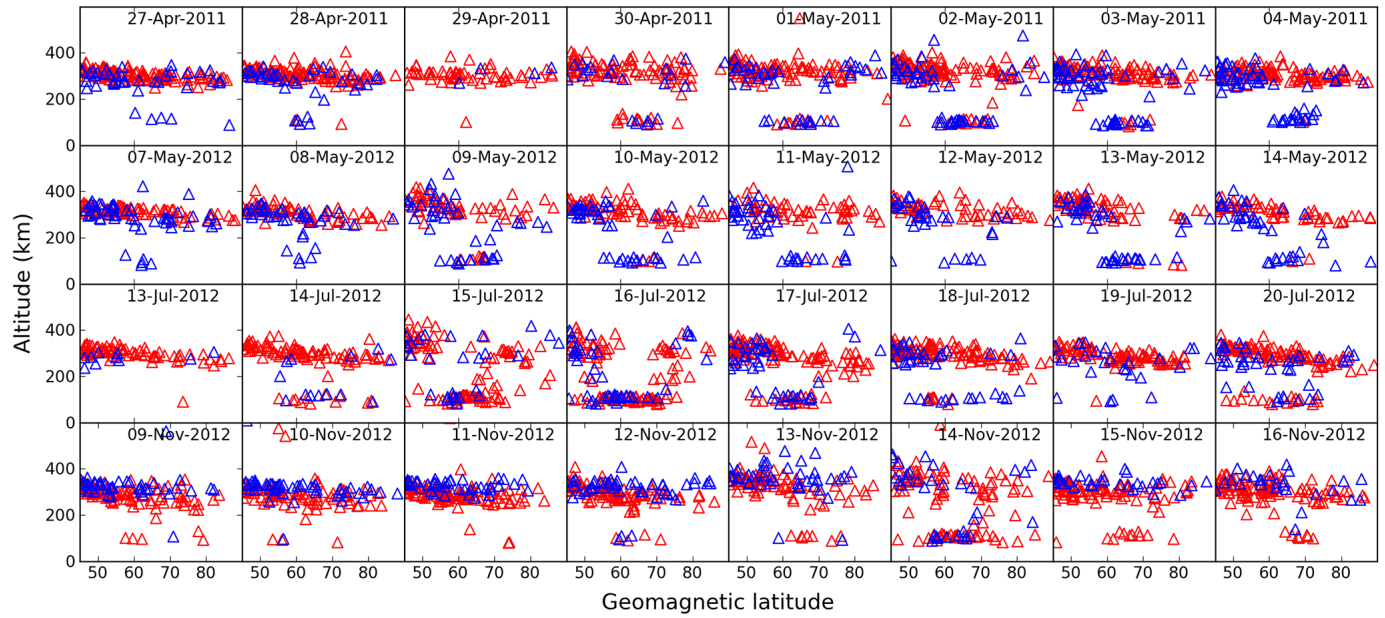

Figure 3. Altitude of peak electron density for the four storm periods studied in this paper. MLT range is 21:00-05:00. All profiles within the MLT range are plotted for Northern (red) and Southern (blue) hemispheres.
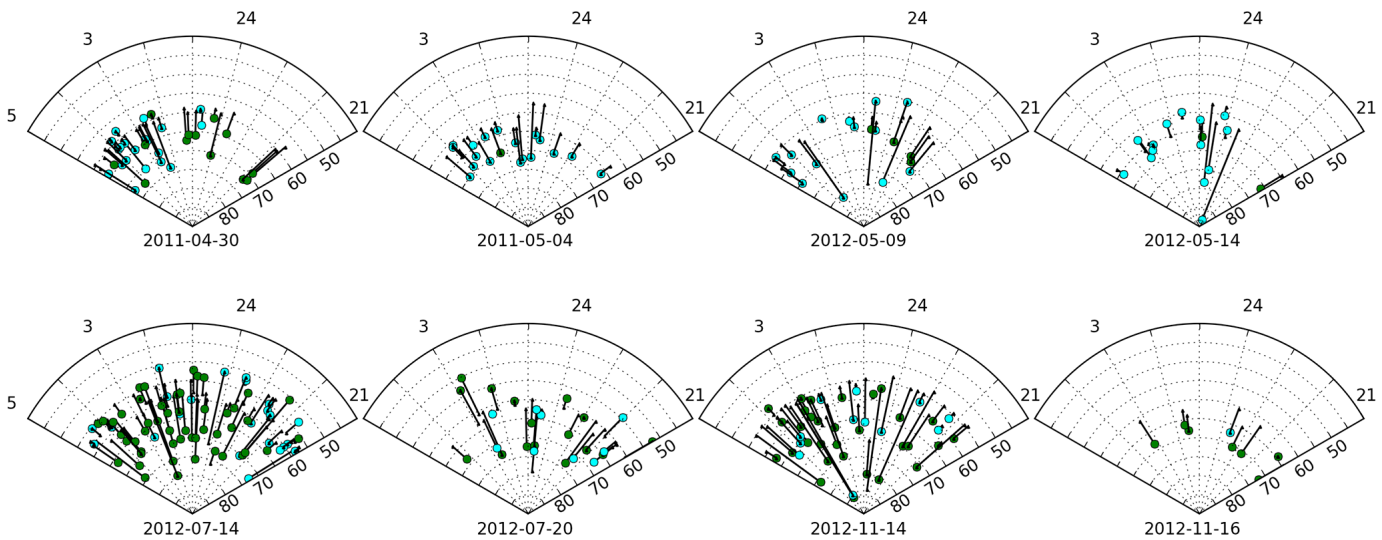

Figure 4. E layer dominant electron density profile locations versus magnetic latitude and MLT for selected days in the four storm periods. MLT range is 21:00-05:00. Green circles are Northern Hemisphere. Cyan circles are Southern Hemisphere. A line connects each circle to the climatological equatorward boundary of the auroral oval, indicated as a black triangle. The boundary is determined based on the IRI2012 model computation (Bilitza et al., 2014; Zhang and Paxton, 2008).

nighttime local times when solar-induced $\mathrm{F}$ region ionization that peaks at altitudes above $200 \mathrm{~km}$ is not present (see Fig. 5 of Mayer and Jakowski, 2009).

The distribution of the height of electron density maximum $h_{\max }$, which is a value made available in the CDAAC profiles, is shown in Fig. 3 for the four storm periods of interest. For each storm we show several days surrounding the storm period, for the magnetic local time (MLT) range of 21:00-05:00 (night sector) in both the Northern (red) and Southern (blue) hemispheres. The increase in precipitation during the storm phases (see Fig. 2 for storm days) is evident in these plots for both hemispheres. The quantity of profiles showing precipitation signature (as ELDI) is significantly higher for the more-intense ICME-generated storms (bottom two panels of Fig. 3) than for the HSSs (top two panels of Fig. 3). Increases in precipitation tend to occur later in the storm phase for the HSSs than for the ICMEs. Additional discussion is included in Sect. 4.

Figure 4 shows the locations of the profiles in Fig. 3 for which the electron density maximum occurs below $200 \mathrm{~km}$, i.e., the profiles showing significant precipitation. Two days for each storm are shown: the day when the maximum number of precipitation events occurs and a day in the following recovery period. Magnetic local time is restricted to 21:00 05:00 MLT, as in Fig. 3. Northern Hemisphere cases are in green; Southern Hemisphere is cyan. Also shown is the location of the climatological equatorward boundary of the auroral oval corresponding to each profile as determined by the model of Zhang and Paxton (2008), which has been incorporated into the 2012 version of the International Reference Ionosphere (Bilitza et al., 2014). This boundary is determined by auroral images from the SUSSI series of satellites 


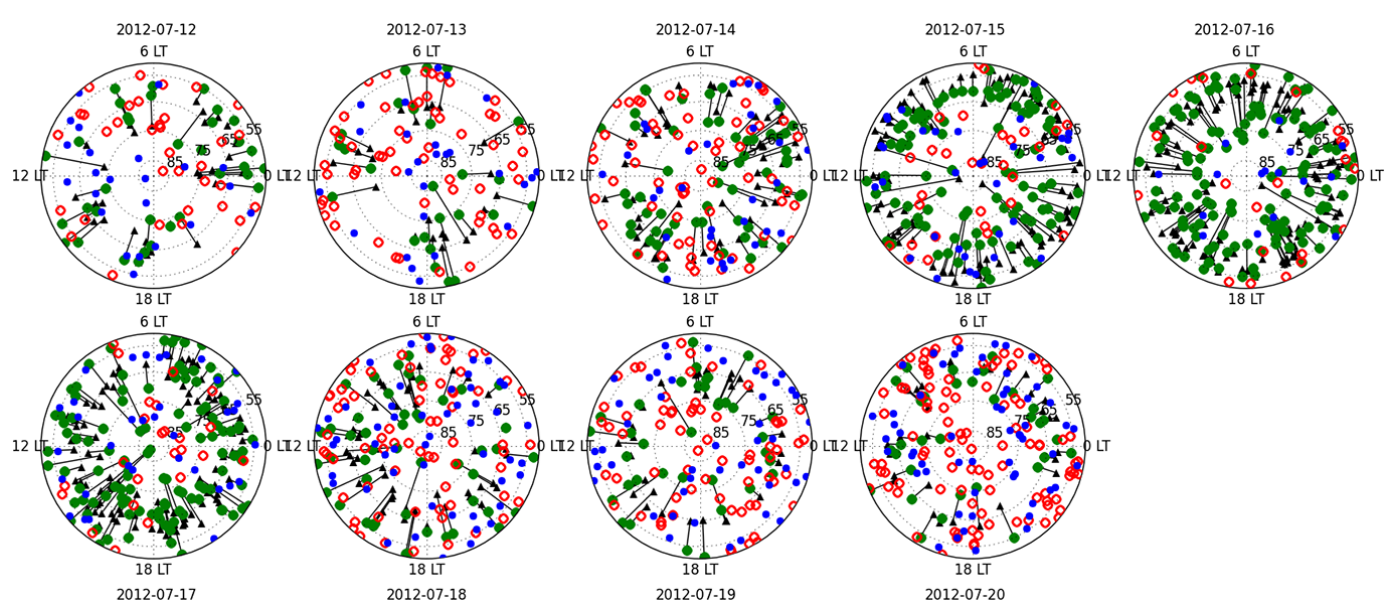

Figure 5. Locations of COSMIC electron density profiles for the July 2012 storm period in MLT/geomagnetic latitude coordinates for the Northern Hemisphere. Green circles indicate the presence of a E region density layer, open red circles indicate no layer present, and blue circles are ambiguous. The climatological boundary from IRI2012 is indicated as in Fig. 4.

as a function of the $K p$ geomagnetic index. The equatorward boundary calculated using the location and time of each profile is indicated by the small black triangles connected by a line to the profile locations. Generally, the location of the climatological auroral boundary is at, or equatorward of, the profile locations for the high-speed stream storms. However, for the CME event in July 2012, several of the profiles are at lower latitudes than the equatorward boundary. This is further discussed in Sect. 4.

The width in geomagnetic latitude of the region where precipitation occurs is generally in the range $60-75^{\circ}$, which is consistent with measurements and models of auroral zone extent (Newell et al., 2002). The climatology was derived for the Northern Hemisphere, but we have applied it to both hemispheres, assuming hemispheric conjugacy.

The situation is somewhat more complex for one of the CME-driven events studied here. For the July 2012 event, there is a systematic underestimation by the model of the precipitation's equatorward boundary during the storm commencement on 14 July 2012, even though the equatorward boundary moves to lower latitudes as the storm intensifies (see Fig. 5). Lower-latitude precipitation also occurs during the storm's recovery phase, as discussed in more detail in Sect. 4.

\section{Case Study: July 2012 CME}

A more detailed analysis is presented of the July 2012 CME event. We wish to remove the restriction to nighttime local times in order to consider all local times for latitudes above $50^{\circ}$. For daytime local times, height of maximum electron density is not a robust indicator of particle precipitation because solar ionizing radiation often creates larger electron densities in the F2 layer than precipitation-induced densities near the E layer (Mayer and Jakowski, 2009).
Rather than develop an automated algorithm, which was not straightforward, we visually inspected profiles to discern the presence of enhanced electron density layers at $\mathrm{E}$ region altitudes. All profiles above $50^{\circ}$ in the Northern Hemisphere for the period 12-20 July 2012 (a total of 1383 profiles) were viewed. The manually inspected profiles were categorized as follows: no evidence of precipitation, clear evidence of E layer density enhancement (assumed due to precipitation) and "ambiguous". The precipitation signature was a single electron density enhancement layer that could be identified against the background density. The layer altitude had to be below $200 \mathrm{~km}$. We note that the absence of a clear density enhancement layer does not indicate that there is no precipitation occurring but rather that the additional electron density due to precipitation is not sufficiently increased over the background to be clearly visible as an enhancement layer in the COSMIC profile. However, when precipitating particle fluxes are not sufficient to create a clear E layer density peak, they are likely not changing the conductivity very much. Some of the electron density profiles showed considerable variability throughout the range of altitudes, which could mask the precipitation signature.

The results for the period 14-20 July 2012 are shown in Fig. 5. A marker is placed in a geomagnetic latitude-MLT coordinate system for each electron density profile. Marker color indicates the presence or absence of scintillation. Open red circles indicate profiles that show no evidence of an enhanced $\mathrm{E}$ layer density. Green circles indicate clear evidence of a density enhancement layer below $200 \mathrm{~km}$ altitude. Small blue circles are ambiguous, for example: data are not available below $150 \mathrm{~km}$ altitudes, or the profile is irregular. Also shown, as black triangles, are the climatological equatorward boundaries of precipitation from the Zhang/Paxton model used in IRI 2012. 
The fraction of profiles displaying precipitation increases significantly during the storm main phase on 15 July. This continues through 17 July, whereas on 18 July the number of profiles showing E layer density enhancement reduces significantly and remains low. On the 3 most active days (15, 16 and 17 July), there is little evidence of a preferred local time for precipitation signatures. Precipitation patterns and their significance are further discussed in Sect. 4.

\section{Discussion}

The science question addressed in this paper is whether COSMIC data can improve knowledge of how conductances change during geomagnetic storms. Such improved knowledge could have a significant impact on the accuracy and utility of global coupled models of the magnetospherethermosphere-ionosphere. Since Pedersen and Hall conductances are calculated based on electron density (Kelley, 2009), data that bear directly on conductances have been limited. Global circulation models of the thermosphereionosphere have used a variety of climatological approaches to estimate conductances. A widely used method of obtaining conductance patterns is based on the work of Fuller-Rowell and Evans (1987). Satellite measurements of precipitation, e.g., by the NOAA satellites (Asikainen and Mursula, 2013), are the input for selecting different conductance patterns. By the nature of this and similar approaches, conductances are approximate and could be refined considerably with direct measurements of electron density. COSMIC provides this possibility.

The relationship between particle properties measured by satellites and conductance patterns in the ionosphere involves multiple assumptions. Robinson et al. (1987) used the assumption of Maxwellian energy distributions of precipitating electrons to derive a relationship between height-integrated conductivity and measured precipitation properties. They found that this relationship is strongly dependent on the spectrum of electron energies. Recent work by Sheng et al. (2014) has revealed significant differences between measured conductance estimates using COSMIC and those estimated using TIE-GCM, a community general circulation model of the coupled thermosphere-ionosphere. Their focus was the ratio of $\mathrm{E}$ to $\mathrm{F}$ layer conductances (height-integrated within each layer), a key ratio that determines how the thermosphere temperature and composition is modified by electrodynamics. This study encompassed several seasons over the years 2008-2011 and was focused on geomagnetically quiet periods. It is reasonable to suggest that disturbed periods will show similar or larger discrepancies between COSMIC measurements and climatologies.

Other indirect methods of obtaining conductances have relied on auroral imagery (Aksnes et al., 2005). However, auroral images are challenged during daytime local times due to interference from dayglow. Multiple assumptions regarding atmospheric properties and how UV emission is related to electron energy spectra are required. COSMIC conductivity measurements have the potential to be more direct and add significantly to our understanding of conductance changes during geomagnetic storms.

Figure 3 shows the altitudes of the peak electron density on a daily basis for the four storm intervals for nighttime MLT. Increased precipitation, whose signature is peak electron density altitudes near $200 \mathrm{~km}$ versus near $350 \mathrm{~km}$, during the magnetic storm periods (see Fig. 2) is clear in these plots. Whereas the ICME storms show a greater fraction of profiles with precipitation signatures during the most intense days, the numbers of profiles showing precipitation tends to be more consistent over time for the HSS storms. This may be associated with continuous driving of the magnetosphere, associated with HILDCAAs, for these HSS storms. Recent research suggests that HILDCAAs are always associated with relativistic precipitation (Hajra et al., 2014). Our limited study suggests that it is worth pursuing the hypothesis that long-duration auroral geomagnetic activity brings a steady stream of precipitating particles into the high-latitude E region.

A notable feature of the two HSS storms is the strong asymmetry of response between the Northern and Southern hemispheres. Both storms occurred in the northern spring season. The 2011 storm starts with approximately equal numbers of ELDI profiles in both hemispheres but ends on 4 May 2011 with nearly all ELDI profiles in the Southern Hemisphere. The May 2012 storm shows several days with predominantly southern hemispheric ELDI profiles. Since the formation of ELDI depends on the magnitude of the F region peak density, solar zenith angle considerations may play a role in the observed hemispheric asymmetry: ELDI requires larger E region density enhancements in the Northern, more sunlit, Hemisphere. However, solar illumination considerations cannot fully explain the observed asymmetry since it occurs consistently at a wide range of geomagnetic latitudes, but the solar illumination hemispheric differences decrease with latitude. Also, the asymmetry varies throughout the storm whereas solar illumination will remain constant over the storm period.

Two causes of auroral hemispheric asymmetry are generally cited in the literature: season and orientation of the interplanetary magnetic field (see Newell et al., 2010, and Østgaard and Laundal, 2012, respectively). Aurora tend to be more intense in the less sunlit hemisphere due to the lower conductivity that increases certain aspects of magnetosphere-ionosphere coupling. That would tend to favor what is observed, which is more ELDI profiles in the Southern Hemisphere. Conversely, a cause of the asymmetry due to the interplanetary magnetic field (IMF) is harder to justify due to the very large fluctuations in the IMF for high-speed streams. In fact, for the May 2012 event the orientation of IMF, despite the fluctuations, changes between 13 and 14 May. For both days (Fig. 2b), $B_{z}$ is predominantly negative (southward). On 13 May, $B_{y}$ rotates from negative 
to positive, and then transitions sharply back to negative on 14 May. Despite this significant rotation of the magnetic field vector, the predominance of Southern Hemisphere precipitation does not vary between these 2 days, suggesting IMF rotation may be a less significant factor than season. More definitive conclusions regarding hemispheric asymmetry is beyond the scope of this paper.

The MLT/latitude locations of the ELDI profiles are shown in Fig. 4 for selected days. The range of MLT is 21:00-05:00, as with Fig. 3. Northern Hemisphere profiles are in green, and Southern Hemisphere profiles are in cyan. The locations of the climatological equatorward boundary are shown as black triangles, connected by a line to the profile location. The climatology reinforces our interpretation of the ELDI profiles as caused by precipitation, since in most cases the profiles lie poleward or near the climatological lower-latitude auroral boundary. An exception appears to be for CME storm starting on 14 July 2012, where a significant number of the profiles are equatorward of the boundary by about $5-10^{\circ}$. Considering the correlation between ELDI profiles and geomagnetic activity, we interpret these lower-latitude profiles as reflecting a limitation of the climatology rather than an indicator that precipitation is not involved in creating the ELDI.

The candidate nighttime precipitation signatures follow the geomagnetic storm evolution, with the signatures increasing and decreasing on a daily basis in concert with the geomagnetic storm intensity. Since the nighttime precipitation was considered only if the $\mathrm{E}$ layer density exceeds density in the $\mathrm{F}$ layer, there are likely additional precipitation signatures in the data that are not shown in Figs. 3 and 4. The time history of profiles that display $\mathrm{E}$ region electron density maxima, and their locations relative to the estimated equatorward boundary of the auroral oval, are consistent with the profiles being reliable indicators of energetic electron precipitation. Our results suggest that COSMIC electron density profiles can provide valuable information on the distribution of conductance changes due to precipitation during geomagnetic storms, thus contributing to a long-standing issue in magnetosphere-ionosphere coupling. The magnitude of the conductance changes can be estimated also, particularly for profiles with more prominent $\mathrm{E}$ layer density increases such as the ELDI profiles.

Figure 5 shows a more detailed analysis of the July 2012 CME storm in the Northern Hemisphere. For this case, we consider all magnetic local times and latitudes down to $50^{\circ}$ magnetic latitude. For these profiles, we did not require the maximum electron density to be at E layer altitudes. We used visual inspection of profiles to determine the presence of a density enhancement layer below $200 \mathrm{~km}$ altitude. Profiles with precipitation signatures are indicated by the green circles in Fig. 5. Open red circles indicate no precipitation signature, and blue circles constitute "ambiguous" cases.

Auroral storm activity began on 14 July (Fig. 2c), although the magnetic storm (Dst, ring current) had not yet entered the main phase. The number of precipitation events has clearly begun to increase on the 14th (Fig. 5) relative to the 2 previous quiet days. A predominance of the precipitation cases is on the nightside.

Significantly enhanced precipitation is observed on days 15-17 July, which encompasses the main phase of the storm and the start of the prolonged recovery phase (note that a smaller new storm is initiated on 17 July by the $B_{z}$ southward turning). Enhanced precipitation seems to have largely decreased by 18 July, deep into the recovery phase.

A notable feature of the storm-time precipitation is its nearly uniform distribution in magnetic local time. Further analysis is required to determine how the impact of the precipitation, as measured by the increased E layer electron densities, is distributed in MLT. Determining impact is complicated by the fact that COSMIC electron density profiles in the $\mathrm{E}$ region are often not accurate to better than $20 \%$, and errors can exceed $100 \%$, due to horizontal plasma gradients affecting the Abel retrievals (Yue et al., 2010). Nevertheless it is clear that precipitation signatures are not confined to the nightside.

The uncertainty of $E$ layer electron densities affects how well ionospheric conductivities can be calculated using radio occultation data, which depend linearly on the electron density (Sheng et al., 2014). The importance of ionospheric conductivities for understanding magnetosphere-ionosphere coupling suggests that further work should be focused on reducing electron density uncertainty for high-latitude profiles.

The characteristics of E region errors in radio occultation are not likely to produce false positive identifications of precipitation using the criteria we are applying. Due to the nature of the retrieval process (Hysell, 2007; Yue et al., 2010; Hajj and Romans, 1998), retrieval errors will not introduce a single electron density enhancement layer of the kind we are using to identify precipitation. The background $\mathrm{E}$ region electron density, defined as the smoothly decreasing component, could certainly be in error. If overestimated, errors in the background might mask a small precipitation enhancement layer. As stated in Sect. 3, multiple precipitation layers were excluded from identification. Thus, we are not subject to false positives due to noisy retrievals as described by Hysell (2007). When excess noise was visible in the E region, we denoted this as an "ambiguous" case. The number of ambiguous cases is not overwhelming our analysis (Fig. 5).

Another notable feature of the storm-time precipitation is its latitude relative to the climatological equatorward boundary. An increase of precipitation signatures equatorward of the boundary is seen on storm days 16-17 July relative to 15 July. This may indicate that COSMIC data are capable of contributing to refined climatologies when combined with existing data sources such as SSUSI and GUVI, from which the climatology is derived. On average, the climatological auroral boundary is most equatorward on 15 July, when the main storm phase occurs, and then retreats somewhat poleward during the recovery phase on 16-17 July. However, the data show a different pattern of largely maintaining the equa- 


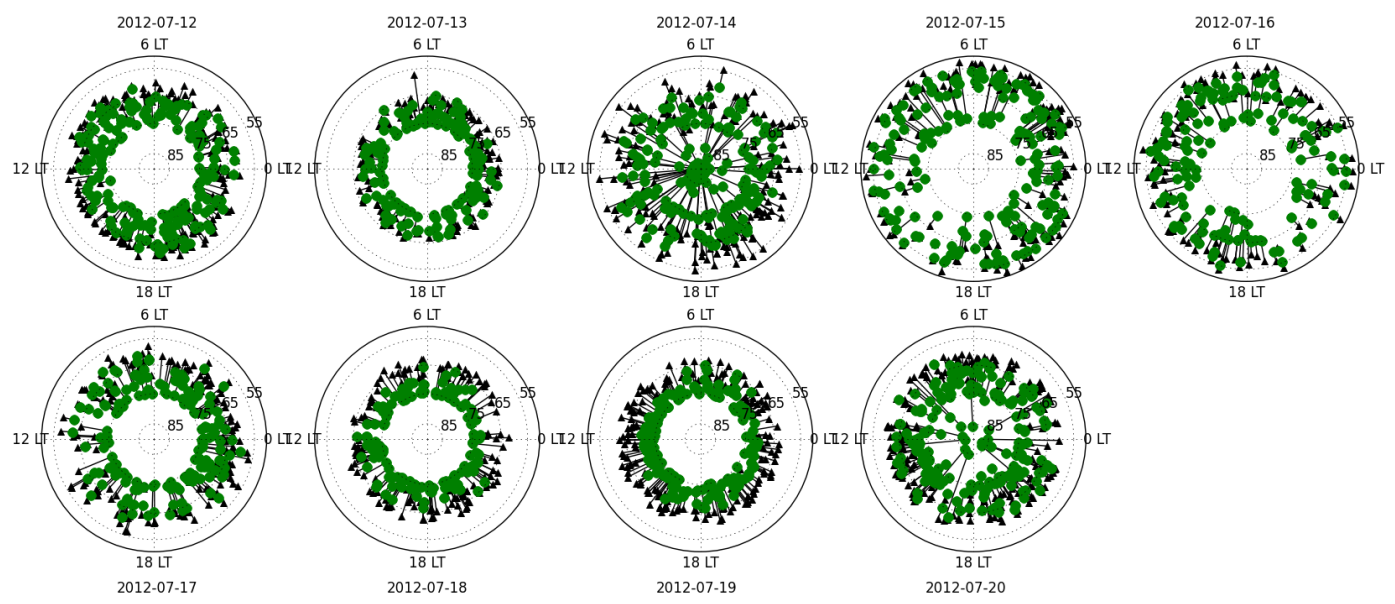

Figure 6. Green circles are auroral boundary locations (decimated) from the SSUSI sensor onboard DMSP satellite F18. The climatological boundary from IRI2012 is indicated as in Fig. 4.

torward extent of the precipitation during the recovery phase compared to the main phase. Persistently increased plasma at low $\mathrm{L}$ shells during the recovery is not surprising and could be the source of the lower-latitude precipitation. It is not clear why the climatology does not capture this effect.

These results are compared with the auroral equatorward boundary product from the SSUSI ultraviolet sensor of the Defense Meteorological Satellite Program (DMSP) satellite F18 (Paxton et al., 2002; http://ssusi.jhuapl.edu). In Fig. 6 we plot the boundary product in a form similar to Fig. 5. The SSUSI data have been decimated so as not to overwhelm the plot and to clearly reveal a dynamically varying auroral region derived from the SSUSI UV imager onboard DMSP. The boundary is based on electron flux values exceeding a threshold of $0.2 \mathrm{ergs} \mathrm{s}^{-1} \mathrm{~cm}^{-2}$ in images that span the equatorward edge of the oval during nighttime (i.e., there are no flux values larger than the threshold more equatorward than the boundary). Dayside boundary locations are based on the Zhang and Paxton GUVI model (2008), which takes the nightside measurements into account. Auroral boundary values in excess of $75^{\circ}$ latitude are likely due to artifacts in the data (see Jones, 2014).

The SSUSI data confirm that, during quiet time, the highlatitude COSMIC E region density layers often fall outside the auroral zone when they appear. During disturbed days starting on 14 July, the equatorward expansion of the auroral oval is largely consistent between COSMIC and SSUSI data until 17 July and after, during the storm's recovery phase (and after a 17 July substorm captured by the Dst index). On 17 July in particular, the SSUSI nightside auroral boundary has retreated to latitudes generally poleward of $65^{\circ}$ geomagnetic latitude, whereas the COSMIC electron density layers are often found equatorward of that, particular on the dayside where the SSUSI data are more model based. On 18 July, conditions are similar to quiet time for the SSUSI auroral boundary.
Comparisons were made with online runs of the Ovation auroral model to assess how COSMIC data sample the poleward boundary of the oval. Ovation is a climatological model of the location of the auroral oval and the intensity of auroral precipitation (Newell et al., 2002). It also accepts real-time data, primarily from the DMSP series of satellites (Hardy et al., 1985). Although Ovation output was not available continuously for the July 2012 storm period, output was available several times per day. We used the latitude extent of the aurora from Ovation, and its temporal changes, to gain further insight into the COSMIC data.

Throughout the 15-17 July storm period, the Ovation oval is mostly confined to latitudes $60-75^{\circ}$ magnetic. Precipitation intensities are more intense for nighttime MLT. The Ovation boundary dips equatorward of $60^{\circ}$ during nighttime on 15 July but stays equatorward of $60^{\circ}$ on the dayside, in contrast to the Zhang/Paxton model which often reaches values less than $55^{\circ}$. The COSMIC data corroborate that the nightside precipitation extends more equatorward than on the dayside for the 15th. On the poleward boundary, there are a few COSMIC events poleward of $75^{\circ}$.

The poleward boundary of Ovation moves somewhat more northward on the 16th compared to the 15th. COSMIC data seem to show agreement with this qualitative feature in that there are a higher fraction of profiles with precipitation signature poleward of $75^{\circ}$ on 16 than 15 July. Thus, the recovery phase seems to bring precipitation to higher latitudes. We note that several COSMIC profiles are poleward of the Ovation boundary on 16 July.

The equatorward boundary of Ovation during the recovery phase on 16 July moves poleward compared to 15 July, as would generally be expected during the recovery phase. However, the COSMIC data tend to have a higher fraction of lower-latitude precipitation events on the 16th, as noted earlier. Thus, there is general disagreement between the COSMIC data and the two climatologies: COSMIC suggests in- 
creased precipitation at lower latitudes during this part of the recovery phase, whereas the models suggest poleward retreat of the lower-latitude boundary.

The recovery phase is interrupted on 17 July by a significant southward turning of IMF $B_{z}$ that may be due to a secondary magnetic cloud feature that follows the first larger magnetic cloud (Fig. 2c). The Dst index stops recovering and indicates an increased ring current in response to this secondary cloud. AE increases also. The COSMIC data show a significant quantity of precipitation events evenly distributed in local time, although a lower fraction of COSMIC profiles show precipitation signatures on 17 versus 16 July. The latitudinal extent of precipitation events is often equatorward of the Zhang/Paxton boundary, a feature common between 17 and 16 July. A significant number of precipitation events are equatorward of $60^{\circ}$. The Ovation model runs show an equatorward boundary significantly above $60^{\circ}$ except at early UT on the dayside when the AE index is increasing on 17 July. We do not show timing for the precipitation profiles, but the significant number of dayside profiles showing precipitation equatorward of $60^{\circ}$ suggests that this storm brings lowerlatitude precipitation than is typically the case.

Ovation runs for 17 July extend the poleward extent of the oval to near $80^{\circ}$ latitude, which is significantly higher latitude than the previous 2 storm days. This appears to be based on the DMSP measurements used by Ovation. COSMIC precipitation signatures are observed poleward of $80^{\circ}$, which barely occurs in the Ovation model. There are a number of "ambiguous" events at these higher latitudes. If these were actual precipitation events, the high-latitude extent of the 17 July storm precipitation would be clearly the highest of all 3 active days, according to COSMIC data. The COSMIC data are suggestive regarding high-latitude precipitation on 17 July.

On 20 July, five profiles out of 15 are at or below $60^{\circ}$ magnetic latitude, which is quite low considering late recovery phase of the storm. However, there was additional solar wind activity on this day, clearly indicated by a proton density increase at approximately 01:00 UT. This did not lead to a geomagnetic storm in the classic sense (Dst decrease) but did cause significant auroral currents as indicated by AE. We speculate here that the fluctuating interplanetary magnetic fields led to precipitation occurring at lower latitudes than would be expected based on the value of the Dst index. COSMIC data may have detected this. Since the Dst index indicates large scale magnetospheric convection that builds up the ring current, either the hot plasma feeding the ring current was absent or large-scale convection was absent on 20 July, even though significant auroral currents and lowerlatitude precipitation did occur. This is an interesting feature deserving of further study.

\section{Summary and conclusions}

Ionospheric conductance changes during storms remain an important topic due to its influence on the thermosphereionosphere both regionally and globally. Global circulation models of the thermosphere-ionosphere suggest significant dependencies on precipitation patterns and the details of high-latitude energy deposition. We have used COSMIC electron density profiles to analyze the presence of enhanced electron density layers in the $\mathrm{E}$ region and determine whether these layers are consistent with ionization from energetic particle precipitation from the magnetosphere during geomagnetic disturbances. We have examined two types of ionization signature: nighttime signatures where the maximum electron density occurs at altitudes below the ionospheric $\mathrm{F}$ layer and evidence of individual precipitation layers at altitudes below the $\mathrm{F}$ region.

We considered two storms (in 2011 and 2012) that began when CIRs in the solar wind were formed by high-speed stream interaction with the slow solar wind. Notable features of nighttime E-region-dominant ionosphere profiles for these two events are increased precipitation that continued for several (5-6) days as the AE index remained elevated; and a strong hemispheric asymmetry, favoring more precipitation in the Southern Hemisphere particularly several days following storm initiation.

We considered two CME-originating storms, both in 2012, which showed larger numbers of daily E-region-dominant profiles on the peak days than the CIR-HSS storms. For the July 2012 storm, the number of E-region-dominant profiles was large also during the storm recovery phase. A more detailed study of the July 2012 CME storm relaxed the requirement of peak density in the $\mathrm{E}$ region to consider all electron density profiles with evidence of an enhanced $\mathrm{E}$ region layer due to precipitation. Precipitation signatures were found nearly uniformly distributed in local time for the 3 storm days 15-17 July, which includes the main phase and 2 days of recovery phase. On 16-17 July, precipitation was often observed equatorward of the climatological auroral boundary. On 17 July in particular, precipitation from COSMIC was often equatorward of the auroral boundary derived from the F18 DMSP satellite SSUSI sensor. Very-highlatitude precipitation was observed also, although at a lower incidence rate. This bears further study in light of recent research by Huang et al. (2014) that polar cap energy deposition can be significant during storms.

Despite concerns of $\mathrm{E}$ region electron density accuracy from COSMIC retrievals, these results suggest that, if care is exercised, COSMIC data from the $\mathrm{E}$ region can be used to learn about conductivity changes during geomagnetic storms in the auroral and polar cap regions. COSMIC profiles with enhanced electron density layers are consistent with expected characteristics of where and when precipitation occurs. The magnitude of the $\mathrm{E}$ layer ionization above background is probably difficult to determine using COSMIC 
Abel-retrieved profiles alone. Despite this limitation, the broad high-latitude coverage of COSMIC profiles is likely to be an excellent resource for characterizing the high-latitude consequences of MI coupling.

Acknowledgements. This research was carried out at the Jet Propulsion Laboratory, California Institute of Technology, under a contract with the National Aeronautics and Space Administration. We acknowledge the support of the Living With a Star Program, within the Heliophysics Division of NASA's Science Mission Directorate. We gratefully acknowledge the provision of Formosat3/COSMIC electron density profile data from the COSMIC Data Analysis and Archive Center and SSUSI data from the Johns Hopkins University Applied Physics Laboratory. We acknowledge use of NASA/Goddard Space Flight Center's Space Physics Data Facility's OMNIWeb service and OMNI data.

Edited by: J. Y. Liu

\section{References}

Aksnes, A., Amm, O., Stadsnes, J., Østgaard, N., Germany, G. A., Vondrak, R. R., and Sillanpää, I.: Ionospheric conductances derived from satellite measurements of auroral UV and X-ray emissions, and ground-based electromagnetic data: a comparison, Ann. Geophys., 23, 343-358, doi:10.5194/angeo-23-343-2005, 2005.

Arras, C., Wickert, J., Beyerle, G., Heise, S., Schmidt, T., and Jacobi, C.: A global climatology of ionospheric irregularities derived from GPS radio occultation, Geophys. Res. Lett., 35, L14809, doi:10.1029/2008g1034158, 2008.

Asikainen, T. and Mursula, K.: Correcting the NOAA/MEPED energetic electron fluxes for detector efficiency and proton contamination, J. Geophys. Res., 118, 6500-6510, doi:10.1002/jgra.50584, 2013.

Bilitza, D., Altadill, D., Zhang, Y., Mertens, C., Truhlik, V., Richards, P., McKinnell, L.-A., and Reinischm B.: The International Reference Ionosphere 2012 - a model of international collaboration, Journal of Space Weather and Space Climate, 4, A07, doi:10.1051/swsc/2014004, 2014.

Cole, K. D.: Joule heating of upper atmosphere, Aust. J. Phys., 15, 223-235, 1962.

Fong, C.-J., Whiteley, D., Yang, E., Cook, K., Chu, V., Schreiner, B., Ector, D., Wilczynski, P., Liu, T.-Y., and Yen, N.: Space and ground segment performance and lessons learned of the FORMOSAT-3/COSMIC mission: four years in orbit, Atmos. Meas. Tech., 4, 1115-1132, doi:10.5194/amt-4-1115-2011, 2011.

Fuller-Rowell, T. J. and Evans, D. S.: Height-integrated Pedersen and Hall conductivity patterns inferred from the TIROSNOAA satellite data, J. Geophys. Res., 92, 7606-7618, doi:10.1029/JA092iA07p07606, 1987.

Hajj, G. A. and Romans, L. J.: Ionospheric electron density profiles obtained with the global positioning system: results from the GPS/MET experiment, Radio Sci., 33, 175-190, A01306, doi:10.1029/2004ja010701, 1998.
Hajra, R., Tsurutani, B. T., Echer, E., and Gonzalez, W. D.: Relativistic electron acceleration during high-intensity, longduration, continuous AE activity (HILDCAA) events: solar cycle phase dependences, Geophys. Res. Lett., 41, 1876-1881, doi:10.1002/2014g1059383, 2014.

Hardy, D. A., Gussenhoven, M. S., and Holeman, E.: A statisticalmodel of auroral electron-precipitation, J. Geophys. Res., 90, 4229-4248, 1985.

Huang, C. Y., Su, Y. J., Sutton, E. K., Weimer, D. R., and Davidson, R. L.: Energy coupling during the August 2011 magnetic storm, J. Geophys. Res-Space: 119, 1219-1232, doi:10.1002/2013ja019297, 2014.

Hysell, D. L.: Inverting ionospheric radio occultation measurements using maximum entropy, Radio Sci., 42, Rs4022, doi:10.1029/2007rs003635, 2007.

Jones, J. C.: Validation of Auroral Oval Models Using DMSP SSUSI, Poster 318, 11th Conference on Space Weather, Annual Meeting of the American Meteorological Society, Atlanta, GA, February 3, available at: https://ams.confex.com/ams/ 94Annual/webprogram/Paper238878.html (last access: 30 June 2015), 2014.

Kelley, M. C.: The Earth's Ionosphere: Electrodynamics and Plasma Physics, 2nd Edn., Elsevier, New York, USA, 2009.

Lei, J., Syndergaard, S., Burns, A. G., Solomon, S. C., Wang, W., Zeng, Z., Roble, R. G., Wu, Q., Kuo, Y.-H., Holt, J. M., Zhang, S.-R., Hysell, D. L., Rodrigues, F. S., and Lin, C. H.: Comparison of COSMIC ionospheric measurements with ground-based observations and model predictions: preliminary results, J. Geophys. Res., 112, A07308, doi:10.1029/2006ja012240, 2007.

Mayer, C. and Jakowski, N.: Enhanced E layer ionization in the auroral zones observed by radio occultation measurements onboard CHAMP and Formosat-3/COSMIC, Ann. Geophys., 27, 1207-1212, doi:10.5194/angeo-27-1207-2009, 2009.

Newell, P. T., Sotirelis, T., Ruohoniemi, J. M., Carbary, J. F., Liou, K., Skura, J. P., Meng, C.-I., Deehr, C., Wilkinson, D., and Rich, F. J.: OVATION: Oval variation, assessment, tracking, intensity, and online nowcasting, Ann. Geophys., 20, 1039-1047, doi:10.5194/angeo-20-1039-2002, 2002.

Newell, P. T., Sotirelis, T., and Wing, S.: Seasonal variations in diffuse, monoenergetic, and broadband aurora, J. Geophys. Res., 115, A03216, doi:10.1029/2009ja014805, 2010.

Nicolls, M. J., Rodrigues, F. S., Bust, G. S., and Chau, J. L.: Estimating $\mathrm{E}$ region density profiles from radio occultation measurements assisted by IDA4D, J. Geophys. Res., 114, A10316, doi:10.1029/2009ja014399, 2009.

Østgaard, N. and Laundal, K. M.: Auroral asymmetries in the conjugate hemispheres and interhemispheric currents, in: Auroral Phenomenology and Magnetospheric Processes: Earth and Other Planets, edited by: Keiling, A., Donovan, E., Bagenal, F., and Karlsson, T., Geoph. Monog. Series, 197, American Geophysical Union, Washington, USA, 99-111, 2012.

Paxton, L. J., Morrison, D., Zhang, Y., Hyosub, K., Wolven, B., Ogorzalek, B. S., Humm, D. C., and Meng, C. I.: Validation of remote sensing products produced by the Special Sensor Ultraviolet Scanning Imager (SSUSI) - a Far-UV Imaging Spectrograph on DMSP F16, Proc. SPIE 1456, Large Screen Projection, Avionic, and Helmet-Mounted Displays, 154, doi:10.1117/12.45426, 2002. 
Robinson, R. M., Vondrak, R. R., Miller, K., Dabbs, T., and Hardy, D.: On calculating ionospheric conductances from the flux and energy of precipitating electrons, J. Geophys. Res., 92, 2565-2569, doi:10.1029/JA092iA03p02565, 1987.

Rocken, C., Kuo, Y. H., Schreiner, W. S., Hunt, D., Sokolovskiy, S., and McCormick, C.: COSMIC system description, Terr. Atmos. Ocean Sci., 11, 21-52, 2000, http://www.ocean-sci.net/11/21/2000/.

Schreiner, W. S., Sokolovskiy, S. V., Rocken, C., and Hunt, D. C.: Analysis and validation of GPS/MET radio occultation data in the ionosphere, Radio Sci., 34, 949-966, doi:10.1029/1999rs900034, 1999.

Sheng, C., Deng, Y., Huang, Y., and Yue, X.: Height-integrated pedersen conductivity of ionosphere from COSMIC observations, in: CEDAR Workshop, Santa Fe, New Mexico, 24-29 June 2012, Santa Fe, NM, SOLA-07, 2012.

Sheng, C., Deng, Y., Yue, X. A., and Huang, Y. S.: Heightintegrated Pedersen conductivity in both $\mathrm{E}$ and $\mathrm{F}$ regions from COSMIC observations, J. Atmos. Sol.-Terr. Phy., 115, 79-86, doi:10.1016/j.jastp.2013.12.013, 2014.

Stephens, P., Komjathy, A., Wilson, B., and Mannucci, A.: New leveling and bias estimation algorithms for processing COSMIC/FORMOSAT-3 data for slant total electron content measurements, Radio Sci., 46, RS0D10, doi:10.1029/2010rs004588, 2011.

Tsurutani, B. T. and Gonzalez, W. D.: The cause of High-Intensity Long-Duration Continuous AE Activity (HILDCAA) Interplanetary Alfvén-Wave Trains, Planet. Space Sci., 35, 405-412, 1987.
Tsurutani, B. T., Gonzalez, W. D., Gonzalez, A. L. C., Guarnieri, F. L., Gopalswamy, N., Grande, M., Kamide, Y., Kasahara, Y., Lu, G., Mann, I., McPherron, R., Soraas, F., and Vasyliunas, V.: Corotating solar wind streams and recurrent geomagnetic activity: a review, J. Geophys. Res., 111, 1-25, doi:10.1029/2005JA011273, 2006.

Wiltberger, M., Wang, W., Burns, A. G., Solomon, S. C., Lyon, J. G., and Goodrich, C. C.: Initial results from the coupled magnetosphere ionosphere thermosphere model: magnetospheric and ionospheric responses, J. Atmos. Sol.-Terr. Phy., 66, 1411-1423, doi:10.1016/j.jastp.2004.03.026, 2004.

Wu, D. L., Ao, C. O., Hajj, G. A., Juarez, M. D., and Mannucci, A. J.: Sporadic E morphology from GPSCHAMP radio occultation, J. Geophys. Res., 110, A01306, doi:10.1029/2004ja010701, 2005.

Yue, X., Schreiner, W. S., Lei, J., Sokolovskiy, S. V., Rocken, C., Hunt, D. C., and Kuo, Y.-H.: Error analysis of Abel retrieved electron density profiles from radio occultation measurements, Ann. Geophys., 28, 217-222, doi:10.5194/angeo-28-217-2010, 2010.

Zhang, Y. and Paxton, L. J.: An empirical Kp-dependent global auroral model based on TIMED/GUVI FUV data, J. Atmos. Sol.-Terr. Phy., 70, 1231-1242, doi:10.1016/j.jastp.2008.03.008, 2008. 